\title{
A generalized inequality for the polar derivative of a polynomial
}

\section{Gulshan Singh 1*, Wali Mohammad Shah² and Abdul Liman³}

\section{"Correspondence:}

gulshansingh1@rediffmail.com

'Bharathiar University, Coimbatore,

Tamil Nadu 641046, India

Full list of author information is

available at the end of the article

\begin{abstract}
In this paper, we extend a result recently proved by Liman et al. (Complex Anal. Oper. Theory 6:1199-1209, 2012. doi:10.1007/s11785-010-0120-3) to the polar derivative of a polynomial and thereby obtain some more general results for polynomials with restricted zeros.
\end{abstract}

MSC: 30A10; 30C10; 30C15

Keywords: polynomial; inequality; zeros; polar derivative

\section{Introduction and statement of results}

Let $P(z):=\sum_{j=0}^{n} a_{j} z^{j}$ be a polynomial of degree $n$, and let $P^{\prime}(z)$ be its derivative, then

$$
\max _{|z|=1}\left|P^{\prime}(z)\right| \leq n \max _{|z|=1}|P(z)|
$$

Inequality (1) is a famous result due to Bernstein and is best possible with equality holding for the polynomial $P(z)=\lambda z^{n}$, where $\lambda$ is a complex number.

If we restrict ourselves to a class of polynomials having no zeros in $|z|<1$, then the above inequality can be sharpened. In fact, Erdös conjectured and later Lax [1] proved that if $P(z) \neq 0$ in $|z|<1$, then

$$
\max _{|z|=1}\left|P^{\prime}(z)\right| \leq \frac{n}{2} \max _{|z|=1}|P(z)|
$$

As a refinement of (2), Aziz and Dawood [2] proved that if $P(z)$ is a polynomial of degree $n$ having no zeros in $|z|<1$, then

$$
\max _{|z|=1}\left|P^{\prime}(z)\right| \leq \frac{n}{2}\left\{\max _{|z|=1}|P(z)|-\min _{|z|=1}|P(z)|\right\} .
$$

As an improvement of (3), Dewan and Hans [3] proved that if $P(z)$ is a polynomial of degree $n$ having no zeros in $|z|<1$, then for any $\beta$ with $|\beta| \leq 1$ and $|z|=1$,

$$
\begin{aligned}
& \left|z P^{\prime}(z)+\frac{n \beta}{2} P(z)\right| \\
& \leq \frac{n}{2}\left\{\left(\left|1+\frac{\beta}{2}\right|+\left|\frac{\beta}{2}\right|\right) \max _{|z|=1}|P(z)|-\left(\left|1+\frac{\beta}{2}\right|-\left|\frac{\beta}{2}\right|\right) \min _{|z|=1}|P(z)|\right\} .
\end{aligned}
$$


Let $D_{\alpha} P(z)$ denote the polar derivative of the polynomial $P(z)$ of degree $n$ with respect to $\alpha$, then

$$
D_{\alpha} P(z)=n P(z)+(\alpha-z) P^{\prime}(z) .
$$

The polynomial $D_{\alpha} P(z)$ is of degree at most $n-1$ and it generalizes the ordinary derivative in the sense that

$$
\lim _{\alpha \rightarrow \infty} \frac{D_{\alpha} P(z)}{\alpha}=P^{\prime}(z)
$$

As an extension of (1) to the polar derivative, Aziz and Shah ([4], Theorem 4 with $k=1$ ) showed that if $P(z)$ is a polynomial of degree $n$, then for every complex number $\alpha$ with $|\alpha|>1$,

$$
\left|D_{\alpha} P(z)\right| \leq n|\alpha| \max _{|z|=1}|P(z)| \quad \text { for }|z|=1 \text {. }
$$

Inequality (5) becomes equality for $P(z)=a z^{n}, a \neq 0$.

If we divide the two sides of (5) by $|\alpha|$ and let $|\alpha| \rightarrow \infty$, we get inequality (1).

Aziz and Shah [5] proved that if $P(z)$ is a polynomial of degree $n$ that does not vanish in $|z|<1$, then for every complex number $\alpha$ with $|\alpha| \geq 1$,

$$
\max _{|z|=1}\left|D_{\alpha} P(z)\right| \leq \frac{n}{2}\left\{(|\alpha|+1) \max _{|z|=1}|P(z)|-(|\alpha|-1) \min _{|z|=1}|P(z)|\right\}
$$

The estimate (6) is best possible with equality for $P(z)=z^{n}+1$. If we divide both sides of (6) by $|\alpha|$ and make $|\alpha| \rightarrow \infty$, we get inequality (3).

As an improvement and generalization to (6) and (4), Liman et al. [6] recently proved the following theorem.

Theorem 1 If $P(z)$ is a polynomial of degree $n$ that does not vanish in $|z|<1$, then for every complex number $\alpha, \beta$ with $|\alpha| \geq 1,|\beta| \leq 1$ and $|z|=1$,

$$
\begin{aligned}
\left|z D_{\alpha} P(z)+n \beta \frac{|\alpha|-1}{2} P(z)\right| \leq & \frac{n}{2}\left\{\left(\left|\alpha+\beta \frac{|\alpha|-1}{2}\right|+\left|z+\beta \frac{|\alpha|-1}{2}\right|\right) \max _{|z|=1}|P(z)|\right. \\
& \left.-\left(\left|\alpha+\beta \frac{|\alpha|-1}{2}\right|-\left|z+\beta \frac{|\alpha|-1}{2}\right|\right) \min _{|z|=1}|P(z)|\right\} .
\end{aligned}
$$

In this paper, we prove the following more general result which is an extension as well as generalization of Theorem 1 and yields a number of known polynomial inequalities.

Theorem 2 Let $P(z)$ be a polynomial of degree $n$ that does not vanish in $|z|<k, k \leq 1$, then for all real or complex numbers $\alpha_{i}$ with $\left|\alpha_{i}\right| \geq k, k \leq 1, i=1,2, \ldots, t, t \leq n-1$ and for any real or complex number $\beta$ with $|\beta| \leq 1$ and for $|z|=1$,

$$
\begin{aligned}
& \left|z^{t} D_{\alpha_{t}} \cdots D_{\alpha_{2}} D_{\alpha_{1}} P(z)+\beta n(n-1) \cdots(n-t+1)\left\{\frac{\left(\left|\alpha_{1}\right|-k\right)\left(\left|\alpha_{2}\right|-k\right) \cdots\left(\left|\alpha_{t}\right|-k\right)}{(1+k)^{t}}\right\} P(z)\right| \\
& \quad \leq \frac{1}{2} n(n-1) \cdots(n-t+1)\left\{\left(\frac{1}{k^{n}}\left|\alpha_{1} \cdot \alpha_{2} \cdots \alpha_{t}+\beta\left\{\frac{\left(\left|\alpha_{1}\right|-k\right)\left(\left|\alpha_{2}\right|-k\right) \cdots\left(\left|\alpha_{t}\right|-k\right)}{(1+k)^{t}}\right\}\right|\right.\right.
\end{aligned}
$$




$$
\begin{aligned}
& \left.+\left|z^{t}+\beta\left\{\frac{\left(\left|\alpha_{1}\right|-k\right)\left(\left|\alpha_{2}\right|-k\right) \cdots\left(\left|\alpha_{t}\right|-k\right)}{(1+k)^{t}}\right\}\right|\right) \max _{|z|=1}|P(z)| \\
& -\left(\frac{1}{k^{n}}\left|\alpha_{1} \cdot \alpha_{2} \cdots \alpha_{t}+\beta\left\{\frac{\left(\left|\alpha_{1}\right|-k\right)\left(\left|\alpha_{2}\right|-k\right) \cdots\left(\left|\alpha_{t}\right|-k\right)}{(1+k)^{t}}\right\}\right|\right. \\
& \left.\left.-\left|z^{t}+\beta\left\{\frac{\left(\left|\alpha_{1}\right|-k\right)\left(\left|\alpha_{2}\right|-k\right) \cdots\left(\left|\alpha_{t}\right|-k\right)}{(1+k)^{t}}\right\}\right|\right) \min _{|z|=k}|P(z)|\right\} .
\end{aligned}
$$

Remark Theorem 1 is a special case of Theorem 2 when we take $t=k=1$.

If we take $t=1$ in Theorem 2, we get the following corollary.

Corollary 1 If $P(z)$ is a polynomial of degree $n$ that does not vanish in $|z|<k, k \leq 1$, then for all complex numbers $\alpha, \beta$ with $|\alpha| \geq k, k \leq 1,|\beta| \leq 1$, and for $|z|=1$,

$$
\begin{aligned}
\left|z D_{\alpha} P(z)+n \beta \frac{|\alpha|-k}{1+k} P(z)\right| \leq & \frac{n}{2}\left\{\left(\frac{1}{k^{n}}\left|\alpha+\beta \frac{|\alpha|-k}{1+k}\right|+\left|z+\beta \frac{|\alpha|-k}{1+k}\right|\right) \max _{|z|=1}|P(z)|\right. \\
& \left.-\left(\frac{1}{k^{n}}\left|\alpha+\beta \frac{|\alpha|-k}{1+k}\right|-\left|z+\beta \frac{|\alpha|-k}{1+k}\right|\right) \min _{|z|=k}|P(z)|\right\} .
\end{aligned}
$$

If we take $k=1$ in Theorem 2 , we get the following result.

Corollary 2 Let $P(z)$ be a polynomial of degree $n$ that does not vanish in $|z| \leq 1$, then for all real or complex numbers $\alpha_{i}$ with $\left|\alpha_{i}\right| \geq 1, i=1,2, \ldots, t, t \leq n-1$ and for any real or complex number $\beta$ with $|\beta| \leq 1$ and for $|z|=1$,

$$
\begin{aligned}
&\left|z^{t} D_{\alpha_{t}} \cdots D_{\alpha_{2}} D_{\alpha_{1}} P(z)+\beta n(n-1) \cdots(n-t+1)\left\{\frac{\left(\left|\alpha_{1}\right|-1\right)\left(\left|\alpha_{2}\right|-1\right) \cdots\left(\left|\alpha_{t}\right|-1\right)}{2^{t}}\right\} P(z)\right| \\
& \leq \leq \frac{1}{2} n(n-1) \cdots(n-t+1)\left\{\left(\left|\alpha_{1} \cdot \alpha_{2} \cdots \alpha_{t}+\beta\left\{\frac{\left(\left|\alpha_{1}\right|-1\right)\left(\left|\alpha_{2}\right|-1\right) \cdots\left(\left|\alpha_{t}\right|-1\right)}{2^{t}}\right\}\right|\right.\right. \\
&\left.+\left|z^{t}+\beta\left\{\frac{\left(\left|\alpha_{1}\right|-1\right)\left(\left|\alpha_{2}\right|-1\right) \cdots\left(\left|\alpha_{t}\right|-1\right)}{2^{t}}\right\}\right|\right) \max _{|z|=1}|P(z)| \\
&-\left(\left|\alpha_{1} \cdot \alpha_{2} \cdots \alpha_{t}+\beta\left\{\frac{\left(\left|\alpha_{1}\right|-1\right)\left(\left|\alpha_{2}\right|-1\right) \cdots\left(\left|\alpha_{t}\right|-1\right)}{2^{t}}\right\}\right|\right. \\
&\left.\left.-\left|z^{t}+\beta\left\{\frac{\left(\left|\alpha_{1}\right|-1\right)\left(\left|\alpha_{2}\right|-1\right) \cdots\left(\left|\alpha_{t}\right|-1\right)}{2^{t}}\right\}\right|\right) \min _{|z|=1}|P(z)|\right\} .
\end{aligned}
$$

For $\beta=0$ and $t=1$ in Theorem 2, we get the following.

Corollary 3 Let $P(z)$ be a polynomial of degree $n$ that does not vanish in $|z|<k, k \leq 1$, then for any real or complex number $\alpha$ with $|\alpha| \geq k, k \leq 1$,

$$
\max _{|z|=1}\left|D_{\alpha} P(z)\right| \leq \frac{n}{2}\left\{\left(\frac{|\alpha|}{k^{n}}+1\right) \max _{|z|=1}|P(z)|-\left(\frac{|\alpha|}{k^{n}}-1\right) \min _{|z|=k}|P(z)|\right\}
$$

If we take $k=1$ in Corollary 3, then (10) reduces to (6).

By taking $t=1$ in (8), dividing both sides of (8) by $|\alpha|$ and letting $|\alpha| \rightarrow \infty$, we have the following generalization of inequality (4). 
Corollary 4 Let $P(z)$ be a polynomial of degree $n$ that does not vanish in $|z|<k, k \leq 1$, then for any real or complex number $\beta$ with $|\beta| \leq 1$ and $|z|=1$,

$$
\begin{aligned}
\left|z P^{\prime}(z)+\frac{n \beta}{1+k} P(z)\right| \leq & \frac{n}{2}\left\{\left(\frac{1}{k^{n}}\left|1+\frac{\beta}{1+k}\right|+\left|\frac{\beta}{1+k}\right|\right) \max _{|z|=1}|P(z)|\right. \\
& \left.-\left(\frac{1}{k^{n}}\left|1+\frac{\beta}{1+k}\right|-\left|\frac{\beta}{1+k}\right|\right) \min _{|z|=k}|P(z)|\right\} .
\end{aligned}
$$

Taking $\beta=0$ and $k=1$ in Corollary 4 , (11) reduces to (3).

\section{Lemmas}

We require the following lemmas. The first lemma follows from Laguerre's theorem [7, p.52] (see also [8]).

Lemma 1 If all the zeros of the $n$th degree polynomial $P(z)$ lie in a circular region $\mathcal{C}$, and if $\xi$ is any zero of

$$
D_{\alpha} P(z)=n P(z)+(\alpha-z) P^{\prime}(z)
$$

the polar derivative of $P(z)$, then both points $\xi$ and $\alpha$ may not lie outside of $\mathcal{C}$.

By repeated applications of Lemma 1, we get the following result, when the circular region $\mathcal{C}$ is the circle $|z| \leq r$.

Lemma 2 If all the zeros of the nth degree polynomial $P(z)$ lie in $|z| \leq r$ and if none of the points $\alpha_{1}, \alpha_{2}, \ldots, \alpha_{t}$ lie in $|z| \leq r$, then each of the polar derivatives $D_{\alpha_{t}} \cdots D_{\alpha_{2}} D_{\alpha_{1}} P(z)$, $t=1,2, \ldots, n-1$, has all its zeros in $|z| \leq r$.

Lemma 3 If $P(z):=a_{0}+\sum_{j=\mu}^{n} a_{j} z^{j}, 1 \leq \mu \leq n$, is a polynomial of degree $n$ having no zeros in the disk $|z|<k, k \geq 1$, then

$$
k^{\mu}\left|P^{\prime}(z)\right| \leq\left|Q^{\prime}(z)\right| \quad \text { for }|z|=1,
$$

where $Q(z)=z^{n} \overline{P\left(\frac{1}{\bar{z}}\right)}$ and $\frac{\mu}{n}\left|\frac{a_{\mu}}{a_{0}}\right| k^{\mu} \leq 1$.

The above lemma is due to Chan and Malik [9].

Lemma 4 If $P(z):=a_{n} z^{n}+\sum_{j=\mu}^{n} a_{n-j} z^{n-j}$ is a polynomial of degree $n$ having all its zeros in the disk $|z| \leq k \leq 1$, then

$$
\left|Q^{\prime}(z)\right| \leq k^{\mu}\left|P^{\prime}(z)\right| \quad \text { for }|z|=1,1 \leq \mu \leq n,
$$

where $Q(z)=z^{n} \overline{P\left(\frac{1}{\bar{z}}\right)}$.

Proof of Lemma 4. Since all the zeros of $P(z)$ lie in $|z| \leq k \leq 1$, therefore all the zeros of $Q(z)=z^{n} \overline{P\left(\frac{1}{\bar{z}}\right)}$ lie in $|z| \geq \frac{1}{k} \geq 1$. Hence applying Lemma 3 to the polynomial $Q(z):=\bar{a}_{n}+$ 
$\sum_{j=\mu}^{n} \bar{a}_{n-j} z^{j}$, we get

$$
\frac{1}{K^{\mu}}\left|Q^{\prime}(z)\right| \leq\left|P^{\prime}(z)\right|
$$

Hence, inequality (12) follows.

Lemma 5 Let $P(z)=a_{n} z^{n}+\sum_{j=\mu}^{n} a_{n-j} z^{n-j}, 1 \leq \mu \leq n$, be a polynomial of degree $n$ having all its zeros in the disk $|z| \leq k, k \leq 1$, then for every real or complex number $\alpha$ with $|\alpha| \geq k$, $k \leq 1$ and for $|z|=1$,

$$
\left|D_{\alpha} P(z)\right| \geq n\left(\frac{|\alpha|-k^{\mu}}{1+k^{\mu}}\right)|P(z)| \text {. }
$$

Proof of Lemma 5 Let $Q(z)=z^{n} \overline{P\left(\frac{1}{\bar{z}}\right)}$, we have $P(z)=z^{n} \overline{Q\left(\frac{1}{\bar{z}}\right)}$. Then it can be easily verified that

$$
\left|Q^{\prime}(z)\right|=\left|n P(z)-z P^{\prime}(z)\right| \quad \text { for }|z|=1
$$

Since $P(z)$ has all its zeros in $|z| \leq k \leq 1$, by Lemma 4 , we get

$$
\left|Q^{\prime}(z)\right| \leq k^{\mu}\left|P^{\prime}(z)\right| \text { for }|z|=1 \text {. }
$$

This implies

$$
\left|P^{\prime}(z)\right|+\left|Q^{\prime}(z)\right| \leq\left(1+k^{\mu}\right)\left|P^{\prime}(z)\right| \text {. }
$$

Also, for $|z|=1$, by using (13), we have

$$
n|P(z)|=\left|n P(z)+z P^{\prime}(z)-z P^{\prime}(z)\right| \leq\left|n P(z)-z P^{\prime}(z)\right|+\left|P^{\prime}(z)\right|=\left|Q^{\prime}(z)\right|+\left|P^{\prime}(z)\right| .
$$

Using (14) in the above inequality, we get

$$
n|P(z)| \leq\left(1+k^{\mu}\right)\left|P^{\prime}(z)\right|
$$

or

$$
\left|P^{\prime}(z)\right| \geq \frac{n}{\left(1+k^{\mu}\right)}|P(z)| \text { for }|z|=1
$$

For every real or complex number $\alpha$ with $|\alpha| \geq k, k \leq 1$, we have

$$
\left|D_{\alpha} P(z)\right|=\left|n P(z)+(\alpha-z) P^{\prime}(z)\right| \text {. }
$$

Now, by using Lemma 4 and (15), we have

$$
\begin{aligned}
\left|D_{\alpha} P(z)\right| & =\left|n P(z)+(\alpha-z) P^{\prime}(z)\right| \\
& =\left|\alpha P^{\prime}(z)+n P(z)-z P^{\prime}(z)\right|
\end{aligned}
$$




$$
\begin{aligned}
& \geq|\alpha|\left|P^{\prime}(z)\right|-\left|n P(z)-z P^{\prime}(z)\right| \\
& =|\alpha|\left|P^{\prime}(z)\right|-\left|Q^{\prime}(z)\right| \text { for }|z|=1 \\
& \geq|\alpha|\left|P^{\prime}(z)\right|-k^{\mu}\left|P^{\prime}(z)\right| \\
& =\left(|\alpha|-k^{\mu}\right)\left|P^{\prime}(z)\right| \\
& \geq n\left(\frac{|\alpha|-k^{\mu}}{1+k^{\mu}}\right)\left|P^{\prime}(z)\right| \text { for }|z|=1 .
\end{aligned}
$$

This completes the proof of Lemma 5.

Lemma 6 Let $P(z)=a_{n} z^{n}+\sum_{j=\mu}^{n} a_{n-j} z^{n-j}, 1 \leq \mu \leq n$, be a polynomial of degree $n$ having all its zeros in the disk $|z| \leq k, k \leq 1$, then for every real or complex number $\alpha_{i}$ with $\left|\alpha_{i}\right| \geq k$, $k \leq 1, i=1,2, \ldots, t, t \leq n-1$ and for $|z|=1$,

$$
\begin{aligned}
& \left|D_{\alpha_{t}} \cdots D_{\alpha_{2}} D_{\alpha_{1}} P(z)\right| \\
& \quad \geq n(n-1) \cdots(n-t+1)\left\{\frac{\left(\left|\alpha_{1}\right|-k^{\mu}\right)\left(\left|\alpha_{2}\right|-k^{\mu}\right) \cdots\left(\left|\alpha_{t}\right|-k^{\mu}\right)}{\left(1+k^{\mu}\right)^{t}}\right\}|P(z)| .
\end{aligned}
$$

Proof of Lemma 6 If $\left|\alpha_{i}\right|=k$ for at least one $i, 1 \leq i \leq t$, then inequality (16) is trivial. Thus, we assume that $\left|\alpha_{i}\right|>k, k \leq 1$, for all $1 \leq i \leq t$. We proceed by the principle of mathematical induction. The result is true for $t=1$ by Lemma 5 , that is, if $\left|\alpha_{1}\right|>k$, then

$$
\left|D_{\alpha_{1}} P(z)\right| \geq n\left(\frac{\left|\alpha_{1}\right|-k^{\mu}}{1+k^{\mu}}\right)|P(z)|
$$

Now, for $t=2$ and for $\left|\alpha_{1}\right|>k, D_{\alpha_{1}} P(z)$ will be a polynomial of degree at most $n-1$. Since all the zeros of $P(z)$ in $|z| \leq k, k \leq 1$, therefore, by applying Lemma 1 , all the zeros of $D_{\alpha_{1}} P(z)$ lie in $|z| \leq k, k \leq 1$, then using Lemma 5 for the polynomial $D_{\alpha_{1}} P(z)$ of degree at most $n-1$, and for $\left|\alpha_{2}\right|>k$, we have

$$
\left|D_{\alpha_{2}}\left\{D_{\alpha_{1}} P(z)\right\}\right| \geq(n-1)\left(\frac{\left|\alpha_{2}\right|-k^{\mu}}{1+k^{\mu}}\right)\left|D_{\alpha_{1}} P(z)\right|
$$

Combining (17) and (18), we get

$$
\left|D_{\alpha_{2}} D_{\alpha_{1}} P(z)\right| \geq n(n-1)\left\{\frac{\left(\left|\alpha_{1}\right|-k^{\mu}\right)\left(\left|\alpha_{2}\right|-k^{\mu}\right)}{\left(1+k^{\mu}\right)^{2}}\right\}|P(z)| .
$$

So, the result is true for $t=2$. Now, we assume that the result is true for $t=v<n$; that is, for $|z|=1$,

$$
\begin{aligned}
& \left|D_{\alpha_{v}} \cdots D_{\alpha_{2}} D_{\alpha_{1}} P(z)\right| \\
& \quad \geq n(n-1) \cdots(n-v+1)\left\{\frac{\left(\left|\alpha_{1}\right|-k^{\mu}\right)\left(\left|\alpha_{2}\right|-k^{\mu}\right) \cdots\left(\left|\alpha_{v}\right|-k^{\mu}\right)}{\left(1+k^{\mu}\right)^{v}}\right\}|P(z)| .
\end{aligned}
$$

We need to show that the result is true for $t=v+1$.

Now corresponding to an $n$th degree polynomial $P(z)$ whose all zeros lie in the disk $|z| \leq k, k \leq 1$, we construct $D_{\alpha_{v}} \cdots D_{\alpha_{2}} D_{\alpha_{1}} P(z)$ a polynomial of degree at most $n-v$ for all 
real or complex numbers $\alpha_{i}$ with $\left|\alpha_{i}\right| \geq k, k \leq 1, i=1,2, \ldots, v(v<n)$ whose all zeros lie in $|z| \leq k$. Therefore, for $\left|\alpha_{v+1}\right|>k$, by applying Lemma 5 to $D_{\alpha_{v}} \cdots D_{\alpha_{2}} D_{\alpha_{1}} P(z)$, we get

$$
\begin{aligned}
& \left|D_{\alpha_{v+1}}\left\{D_{\alpha_{v}} \cdots D_{\alpha_{2}} D_{\alpha_{1}} P(z)\right\}\right| \\
& \quad \geq(n-v)\left(\frac{\left|\alpha_{v+1}\right|-k^{\mu}}{1+k^{\mu}}\right)\left|D_{\alpha_{v}} \cdots D_{\alpha_{2}} D_{\alpha_{1}} P(z)\right| .
\end{aligned}
$$

Combining (19) and (20), we obtain

$$
\begin{aligned}
& \left|D_{\alpha_{v+1}} D_{\alpha_{v}} \cdots D_{\alpha_{2}} D_{\alpha_{1}} P(z)\right| \\
& \geq n(n-1) \cdots(n-v)\left\{\frac{\left(\left|\alpha_{1}\right|-k^{\mu}\right)\left(\left|\alpha_{2}\right|-k^{\mu}\right) \cdots\left(\left|\alpha_{v+1}\right|-k^{\mu}\right)}{\left(1+k^{\mu}\right)^{v+1}}\right\}|P(z)| .
\end{aligned}
$$

This implies that the result is true for $t=v+1$ and this completes the proof of Lemma 6 .

Lemma 7 Let $P(z)=a_{n} z^{n}+\sum_{j=\mu}^{n} a_{n-j} z^{n-j}, 1 \leq \mu \leq n$, be a polynomial of degree $n$ having all its zeros in the disk $|z| \leq k, k \leq 1$. Then for every real or complex number $\alpha_{i}$ with $\left|\alpha_{i}\right| \geq k$, $k \leq 1, i=1,2, \ldots, t, t \leq n-1$, and for any real or complex number $\beta$ with $|\beta| \leq 1$ and for $|z|=1$,

$$
\begin{aligned}
& \mid z^{t} D_{\alpha_{t}} \cdots D_{\alpha_{2}} D_{\alpha_{1}} P(z)+n(n-1) \cdots(n-t+1) \\
& \quad \times \beta\left\{\frac{\left(\left|\alpha_{1}\right|-k^{\mu}\right)\left(\left|\alpha_{2}\right|-k^{\mu}\right) \cdots\left(\left|\alpha_{t}\right|-k^{\mu}\right)}{\left(1+k^{\mu}\right)^{t}}\right\} P(z) \mid \\
& \geq n(n-1) \cdots(n-t+1) \\
& \quad \times \frac{1}{k^{n}}\left|\alpha_{1} \cdot \alpha_{2} \cdots \alpha_{t}+\beta\left\{\frac{\left(\left|\alpha_{1}\right|-k^{\mu}\right)\left(\left|\alpha_{2}\right|-k^{\mu}\right) \cdots\left(\left|\alpha_{t}\right|-k^{\mu}\right)}{\left(1+k^{\mu}\right)^{t}}\right\}\right| \min _{|z|=k}|P(z)| .
\end{aligned}
$$

Proof of Lemma 7 The result is clear if $P(z)$ has a zero on $|z|=k$, then $m=\min _{|z|=k}|P(z)|=0$. We now suppose that all the zeros of $P(z)$ lie in $|z|<k$, then $m>0$, and we have $m \leq|P(z)|$ for $|z|=k$. Hence, for every $\lambda$ with $|\lambda|<1$, we have $|P(z)|>\left|m \lambda\left(\frac{z}{k}\right)^{n}\right|$ for $|z|=k$. Therefore, it follows by Rouche's theorem that the polynomial $G(z)=P(z)-m \lambda\left(\frac{z}{k}\right)^{n}$ has all its zeros in $|z|<k, k \leq 1$. As $\alpha_{1}, \alpha_{2}, \ldots, \alpha_{t}$ are complex numbers with $\left|\alpha_{i}\right| \geq k, k \leq 1, i=1,2, \ldots, t$, $t \leq n-1$, then by Lemma 2 all the zeros of

$$
\begin{aligned}
& D_{\alpha_{t}} \cdots D_{\alpha_{2}} D_{\alpha_{1}} G(z) \\
& \quad=D_{\alpha_{t}} \cdots D_{\alpha_{2}} D_{\alpha_{1}} P(z)-\lambda \frac{m}{k^{n}}\left\{n(n-1) \cdots(n-t+1) \alpha_{1} \cdot \alpha_{2} \cdots \alpha_{t}\right\} z^{n-t}
\end{aligned}
$$

lie in $|z|<k$. Applying Lemma 6 to the polynomial $G(z)$, we get

$$
\begin{aligned}
& \left|z^{t} D_{\alpha_{t}} \cdots D_{\alpha_{2}} D_{\alpha_{1}} G(z)\right| \\
& \quad \geq n(n-1) \cdots(n-t+1)\left\{\frac{\left(\left|\alpha_{1}\right|-k^{\mu}\right)\left(\left|\alpha_{2}\right|-k^{\mu}\right) \cdots\left(\left|\alpha_{t}\right|-k^{\mu}\right)}{\left(1+k^{\mu}\right)^{t}}\right\}|G(z)|, \quad|z|=1 .
\end{aligned}
$$


Since $z^{t} D_{\alpha_{t}} \cdots D_{\alpha_{2}} D_{\alpha_{1}} G(z)$ has all its zeros in $|z|<k, k \leq 1$, therefore by Rouche's theorem, it follows from inequality (22) that the polynomial

$$
\begin{aligned}
& z^{t} D_{\alpha_{t}} \cdots D_{\alpha_{2}} D_{\alpha_{1}} G(z)+\beta n(n-1) \cdots(n-t+1) \\
& \quad \times\left\{\frac{\left(\left|\alpha_{1}\right|-k^{\mu}\right)\left(\left|\alpha_{2}\right|-k^{\mu}\right) \cdots\left(\left|\alpha_{t}\right|-k^{\mu}\right)}{\left(1+k^{\mu}\right)^{t}}\right\} G(z)
\end{aligned}
$$

has all its zeros in $|z|<1$, where $|\beta|<1$.

By substituting for $G(z)$, we conclude that the polynomial

$$
\begin{aligned}
T(z)= & z^{t} D_{\alpha_{t}} \cdots D_{\alpha_{2}} D_{\alpha_{1}} P(z)+n(n-1) \cdots(n-t+1) \\
& \times \beta\left(\frac{\left(\left|\alpha_{1}\right|-k^{\mu}\right)\left(\left|\alpha_{2}\right|-k^{\mu}\right) \cdots\left(\left|\alpha_{t}\right|-k^{\mu}\right)}{\left(1+k^{\mu}\right)^{t}}\right) P(z) \\
& -\lambda m\left(\frac{z}{k}\right)^{n} n(n-1) \cdots(n-t+1) \\
& \times\left\{\alpha_{1} \cdot \alpha_{2} \cdots \alpha_{t}+\beta\left(\frac{\left(\left|\alpha_{1}\right|-k^{\mu}\right)\left(\left|\alpha_{2}\right|-k^{\mu}\right) \cdots\left(\left|\alpha_{t}\right|-k^{\mu}\right)}{\left(1+k^{\mu}\right)^{t}}\right)\right\}
\end{aligned}
$$

will have no zeros in $|z| \geq 1$. This implies that for every $\beta$ with $|\beta|<1$ and $|z| \geq 1$,

$$
\begin{aligned}
& \mid z^{t} D_{\alpha_{t}} \cdots D_{\alpha_{2}} D_{\alpha_{1}} P(z)+n(n-1) \cdots(n-t+1) \\
& \quad \times \beta\left(\frac{\left(\left|\alpha_{1}\right|-k^{\mu}\right)\left(\left|\alpha_{2}\right|-k^{\mu}\right) \cdots\left(\left|\alpha_{t}\right|-k^{\mu}\right)}{\left(1+k^{\mu}\right)^{t}}\right) P(z) \mid \\
& \geq n(n-1) \cdots(n-t+1) m\left|\frac{z}{k}\right|^{n} \\
& \quad \times\left|\alpha_{1} \cdot \alpha_{2} \cdots \alpha_{t}+\beta\left(\frac{\left(\left|\alpha_{1}\right|-k^{\mu}\right)\left(\left|\alpha_{2}\right|-k^{\mu}\right) \cdots\left(\left|\alpha_{t}\right|-k^{\mu}\right)}{\left(1+k^{\mu}\right)^{t}}\right)\right| .
\end{aligned}
$$

If (24) is not true, then there exists a point $z=\omega$ with $|\omega| \geq 1$ such that

$$
\begin{aligned}
& \mid \omega^{t} D_{\alpha_{t}} \cdots D_{\alpha_{2}} D_{\alpha_{1}} P(\omega)+n(n-1) \cdots(n-t+1) \\
& \quad \times \beta\left(\frac{\left(\left|\alpha_{1}\right|-k^{\mu}\right)\left(\left|\alpha_{2}\right|-k^{\mu}\right) \cdots\left(\left|\alpha_{t}\right|-k^{\mu}\right)}{\left(1+k^{\mu}\right)^{t}}\right) P(\omega) \mid \\
& <n(n-1) \cdots(n-t+1) m\left|\frac{\omega}{k}\right|^{n} \\
& \quad \times\left|\alpha_{1} \cdot \alpha_{2} \cdots \alpha_{t}+\beta\left(\frac{\left(\left|\alpha_{1}\right|-k^{\mu}\right)\left(\left|\alpha_{2}\right|-k^{\mu}\right) \cdots\left(\left|\alpha_{t}\right|-k^{\mu}\right)}{\left(1+k^{\mu}\right)^{t}}\right)\right| .
\end{aligned}
$$

We take

$$
\lambda=\frac{\omega^{t} D_{\alpha_{t}} \cdots D_{\alpha_{2}} D_{\alpha_{1}} P(\omega)+n(n-1) \cdots(n-t+1) \beta\left\{\frac{\left(\left|\alpha_{1}\right|-k^{\mu}\right)\left(\left|\alpha_{2}\right|-k^{\mu}\right) \cdots\left(\left|\alpha_{t}\right|-k^{\mu}\right)}{\left(1+k^{\mu}\right)^{t}}\right\} P(\omega)}{n(n-1) \cdots(n-t+1) m\left(\frac{\omega}{k}\right)^{n}\left(\alpha_{1} \cdot \alpha_{2} \cdots \alpha_{t}+\beta\left\{\frac{\left(\left|\alpha_{1}\right|-k^{\mu}\right)\left(\left|\alpha_{2}\right|-k^{\mu}\right) \cdots\left(\left|\alpha_{t}\right|-k^{\mu}\right)}{\left(1+k^{\mu}\right)^{t}}\right\}\right)}
$$


so that $|\lambda|<1$, and with this choice of $\lambda$, we have $T(\omega)=0$ for $|\omega| \geq 1$ from (23). But this contradicts the fact that $T(z) \neq 0$ for $|z| \geq 1$. For $\beta$ with $|\beta|=1$, (24) follows by continuity. This completes the proof of Lemma 7.

Lemma 8 Let $P(z)=a_{n} z^{n}+\sum_{j=\mu}^{n} a_{n-j} z^{n-j}, 1 \leq \mu \leq n$, be a polynomial of degree $n$. Then for all real or complex numbers $\alpha_{i}$ with $\left|\alpha_{i}\right| \geq k, k \leq 1, i=1,2, \ldots, t, t \leq n-1$, and for any real or complex number $\beta$ with $|\beta| \leq 1$ and for $|z|=1$,

$$
\begin{aligned}
& \mid z^{t} D_{\alpha_{t}} \cdots D_{\alpha_{2}} D_{\alpha_{1}} P(z)+n(n-1) \cdots(n-t+1) \\
& \quad \times \beta\left\{\frac{\left(\left|\alpha_{1}\right|-k^{\mu}\right)\left(\left|\alpha_{2}\right|-k^{\mu}\right) \cdots\left(\left|\alpha_{t}\right|-k^{\mu}\right)}{\left(1+k^{\mu}\right)^{t}}\right\} P(z) \mid \\
& \leq n(n-1) \cdots(n-t+1) \\
& \quad \times \frac{1}{k^{n}}\left|\alpha_{1} \cdot \alpha_{2} \cdots \alpha_{t}+\beta\left\{\frac{\left(\left|\alpha_{1}\right|-k^{\mu}\right)\left(\left|\alpha_{2}\right|-k^{\mu}\right) \cdots\left(\left|\alpha_{t}\right|-k^{\mu}\right)}{\left(1+k^{\mu}\right)^{t}}\right\}\right| \max _{|z|=k}|P(z)| .
\end{aligned}
$$

Proof of Lemma 8 Let $M=\max _{|z|=k}|P(z)|$, if $|\lambda|<1$, then $|\lambda P(z)|<\left|M\left(\frac{z}{k}\right)^{n}\right|$ for $|z|=k$. Therefore, it follows by Rouche's theorem that the polynomial $G(z)=M\left(\frac{z}{k}\right)^{n}-\lambda P(z)$ has all its zeros in $|z|<k, k \leq 1$. As $\alpha_{1}, \alpha_{2}, \ldots, \alpha_{t}$ are complex numbers with $\left|\alpha_{i}\right| \geq k, k \leq 1$, $i=1,2, \ldots, t, t \leq n-1$, then by Lemma 2 all the zeros of

$$
D_{\alpha_{t}} \cdots D_{\alpha_{2}} D_{\alpha_{1}} G(z)=\frac{M}{k^{n}}\left\{n(n-1) \cdots(n-t+1) \alpha_{1} \cdot \alpha_{2} \cdots \alpha_{t}\right\} z^{n-t}-\lambda D_{\alpha_{t}} \cdots D_{\alpha_{2}} D_{\alpha_{1}} P(z)
$$

lie in $|z|<k$. Applying Lemma 6 to the polynomial $G(z)$, we have

$$
\begin{aligned}
& \left|z^{t} D_{\alpha_{t}} \cdots D_{\alpha_{2}} D_{\alpha_{1}} G(z)\right| \\
& \quad \geq n(n-1) \cdots(n-t+1)\left\{\frac{\left(\left|\alpha_{1}\right|-k^{\mu}\right)\left(\left|\alpha_{2}\right|-k^{\mu}\right) \cdots\left(\left|\alpha_{t}\right|-k^{\mu}\right)}{\left(1+k^{\mu}\right)^{t}}\right\}|G(z)|, \quad|z|=1 .
\end{aligned}
$$

Now, if we proceed similarly as in Lemma 7, the result follows.

Lemma 9 Let $P(z)$ be a polynomial of degree $n$, then for all real or complex numbers $\alpha_{i}$ with $\left|\alpha_{i}\right| \geq k, i=1,2, \ldots, t$, and for any real or complex number $\beta$ with $|\beta| \leq 1$,

$$
\begin{aligned}
& \left|z^{t} D_{\alpha_{t}} \cdots D_{\alpha_{2}} D_{\alpha_{1}} P(z)+n(n-1) \cdots(n-t+1) \beta\left\{\frac{\left(\left|\alpha_{1}\right|-k\right)\left(\left|\alpha_{2}\right|-k\right) \cdots\left(\left|\alpha_{t}\right|-k\right)}{(1+k)^{t}}\right\} P(z)\right| \\
& +\mid z^{t} D_{\alpha_{t}} \cdots D_{\alpha_{2}} D_{\alpha_{1}} Q(z)+n(n-1) \cdots(n-t+1) \\
& \times \beta\left\{\frac{\left(\left|\alpha_{1}\right|-k\right)\left(\left|\alpha_{2}\right|-k\right) \cdots\left(\left|\alpha_{t}\right|-k\right)}{(1+k)^{t}}\right\} Q(z) \mid \\
& \leq n(n-1) \cdots(n-t+1)\left\{\frac{1}{k^{n}}\left|\alpha_{1} \cdot \alpha_{2} \cdots \alpha_{t}+\beta\left\{\frac{\left(\left|\alpha_{1}\right|-k\right)\left(\left|\alpha_{2}\right|-k\right) \cdots\left(\left|\alpha_{t}\right|-k\right)}{(1+k)^{t}}\right\}\right|\right. \\
& \left.+\left|z^{t}+\beta\left\{\frac{\left(\left|\alpha_{1}\right|-k\right)\left(\left|\alpha_{2}\right|-k\right) \cdots\left(\left|\alpha_{t}\right|-k\right)}{(1+k)^{t}}\right\}\right|\right\} \max _{|z|=1}|P(z)|, \quad \text { for }|z|=1,
\end{aligned}
$$


where

$$
Q(z):=\left(\frac{z}{k}\right)^{n} \overline{P\left(\frac{k^{2}}{\bar{z}}\right)} .
$$

Proof of Lemma 9 Let $M=\max _{|z|=k}|P(z)|$, then $|P(z)| \leq M$ for $|z| \leq k$. If $\lambda$ is any real or complex number with $|\lambda|>1$, then by Rouche's theorem the polynomial $G(z)=P(z)-\lambda M$ does not vanish in $|z|<k$. Consequently, the polynomial

$$
H(z):=\left(\frac{z}{k}\right)^{n} \overline{G\left(\frac{k^{2}}{\bar{z}}\right)}
$$

has all zeros in $|z| \leq k$ and $|G(z)|=|H(z)|$ for $|z|=k$. Since all the zeros of $H(z)$ lie in $|z| \leq k$, $k \leq 1$, therefore, for $\delta$ with $|\delta|>1$, by Rouche's theorem, all the zeros of $G(z)+\delta H(z)$ lie in $|z| \leq k$. Hence, by taking $\mu=1$ in Lemma 6 and using it, for every real or complex number $\alpha_{i}$ with $\left|\alpha_{i}\right| \geq k, i=1,2, \ldots, t(1 \leq t<n), k \leq 1$, and $|z|=1$, we have

$$
\begin{aligned}
& \left|z^{t} D_{\alpha_{t}} \cdots D_{\alpha_{2}} D_{\alpha_{1}}(G(z)+\delta H(z))\right| \\
& \quad \geq n(n-1) \cdots(n-t+1)\left\{\frac{\left(\left|\alpha_{1}\right|-k\right)\left(\left|\alpha_{2}\right|-k\right) \cdots\left(\left|\alpha_{t}\right|-k\right)}{(1+k)^{t}}\right\}|G(z)+\delta H(z)| .
\end{aligned}
$$

Also, by Lemma 2, all the zeros $D_{\alpha_{t}} \cdots D_{\alpha_{2}} D_{\alpha_{1}}(G(z)+\delta H(z))$ lie in $|z|<k \leq 1$, where $\left|\alpha_{i}\right| \geq$ $k, i=1,2, \ldots, t(1 \leq t<n)$. Therefore, for any $\beta$ with $|\beta|<1$, Rouche's theorem implies that all the zeros of

$$
\begin{aligned}
& z^{t} D_{\alpha_{t}} \cdots D_{\alpha_{2}} D_{\alpha_{1}}(G(z)+\delta H(z)) \\
& \quad+\beta n(n-1) \cdots(n-t+1)\left\{\frac{\left(\left|\alpha_{1}\right|-k\right)\left(\left|\alpha_{2}\right|-k\right) \cdots\left(\left|\alpha_{t}\right|-k\right)}{(1+k)^{t}}\right\}(G(z)+\delta H(z))
\end{aligned}
$$

lie in $|z|<1$.

So, the polynomial

$$
\begin{aligned}
T(z)= & z^{t} D_{\alpha_{t}} \cdots D_{\alpha_{2}} D_{\alpha_{1}} G(z)+\beta n(n-1) \cdots(n-t+1) \\
& \times\left\{\frac{\left(\left|\alpha_{1}\right|-k\right)\left(\left|\alpha_{2}\right|-k\right) \cdots\left(\left|\alpha_{t}\right|-k\right)}{(1+k)^{t}}\right\} G(z) \\
& +\delta\left(z^{t} D_{\alpha_{t}} \cdots D_{\alpha_{2}} D_{\alpha_{1}} H(z)+\beta n(n-1) \cdots(n-t+1)\right. \\
& \left.\times\left\{\frac{\left(\left|\alpha_{1}\right|-k\right)\left(\left|\alpha_{2}\right|-k\right) \cdots\left(\left|\alpha_{t}\right|-k\right)}{(1+k)^{t}}\right\} H(z)\right)
\end{aligned}
$$

will have no zeros in $|z| \geq 1$. Now, using a similar argument as that in the proof of Lemma 7 and taking $\mu=1$, we get for $|z| \geq 1$,

$$
\begin{aligned}
& \mid z^{t} D_{\alpha_{t}} \cdots D_{\alpha_{2}} D_{\alpha_{1}} G(z)+\beta n(n-1) \cdots(n-t+1) \\
& \quad \times\left\{\frac{\left(\left|\alpha_{1}\right|-k\right)\left(\left|\alpha_{2}\right|-k\right) \cdots\left(\left|\alpha_{t}\right|-k\right)}{(1+k)^{t}}\right\} G(z) \mid
\end{aligned}
$$




$$
\begin{aligned}
\leq & \mid z^{t} D_{\alpha_{t}} \cdots D_{\alpha_{2}} D_{\alpha_{1}} H(z)+\beta n(n-1) \cdots(n-t+1) \\
& \times\left\{\frac{\left(\left|\alpha_{1}\right|-k\right)\left(\left|\alpha_{2}\right|-k\right) \cdots\left(\left|\alpha_{t}\right|-k\right)}{(1+k)^{t}}\right\} H(z) \mid .
\end{aligned}
$$

Now,

$$
H(z):=\left(\frac{z}{k}\right)^{n} \overline{G\left(\frac{k^{2}}{\bar{z}}\right)}=\left(\frac{z}{k}\right)^{n} \overline{P\left(\frac{k^{2}}{\bar{z}}\right)}-\bar{\lambda}\left(\frac{z}{k}\right)^{n} M=Q(z)-\bar{\lambda}\left(\frac{z}{k}\right)^{n} M .
$$

On substituting $G(z)$ and $H(z)$ in (26), we obtain the following:

$$
\begin{aligned}
\mid z^{t} D_{\alpha_{t}} \cdots D_{\alpha_{2}} D_{\alpha_{1}} P(z)+\beta n(n-1) \cdots(n-t+1)\left\{\frac{\left(\left|\alpha_{1}\right|-k\right)\left(\left|\alpha_{2}\right|-k\right) \cdots\left(\left|\alpha_{t}\right|-k\right)}{(1+k)^{t}}\right\} P(z) \\
\quad-\lambda M n(n-1) \cdots(n-t+1)\left(z^{t}+\beta\left\{\frac{\left(\left|\alpha_{1}\right|-k\right)\left(\left|\alpha_{2}\right|-k\right) \cdots\left(\left|\alpha_{t}\right|-k\right)}{(1+k)^{t}}\right\}\right) \mid \\
\leq \mid z^{t} D_{\alpha_{t}} \cdots D_{\alpha_{2}} D_{\alpha_{1}} Q(z)+\beta n(n-1) \cdots(n-t+1) \\
\quad \times\left\{\frac{\left(\left|\alpha_{1}\right|-k\right)\left(\left|\alpha_{2}\right|-k\right) \cdots\left(\left|\alpha_{t}\right|-k\right)}{(1+k)^{t}}\right\} Q(z) \\
-\bar{\lambda} M n(n-1) \cdots(n-t+1)\left(\frac{z}{k}\right)^{n} \\
\quad \times\left(\alpha_{1} \cdot \alpha_{2} \cdots \alpha_{t}+\beta\left\{\frac{\left(\left|\alpha_{1}\right|-k\right)\left(\left|\alpha_{2}\right|-k\right) \cdots\left(\left|\alpha_{t}\right|-k\right)}{(1+k)^{t}}\right\}\right) .
\end{aligned}
$$

This implies that

$$
\begin{aligned}
& \left|z^{t} D_{\alpha_{t}} \cdots D_{\alpha_{2}} D_{\alpha_{1}} P(z)+\beta n(n-1) \cdots(n-t+1)\left\{\frac{\left(\left|\alpha_{1}\right|-k\right)\left(\left|\alpha_{2}\right|-k\right) \cdots\left(\left|\alpha_{t}\right|-k\right)}{(1+k)^{t}}\right\} P(z)\right| \\
& \quad-\left|\lambda M n(n-1) \cdots(n-t+1)\left(z^{t}+\beta\left\{\frac{\left(\left|\alpha_{1}\right|-k\right)\left(\left|\alpha_{2}\right|-k\right) \cdots\left(\left|\alpha_{t}\right|-k\right)}{(1+k)^{t}}\right\}\right)\right| \\
& \leq \mid z^{t} D_{\alpha_{t}} \cdots D_{\alpha_{2}} D_{\alpha_{1}} Q(z)+\beta n(n-1) \cdots(n-t+1) \\
& \quad \times\left\{\frac{\left(\left|\alpha_{1}\right|-k\right)\left(\left|\alpha_{2}\right|-k\right) \cdots\left(\left|\alpha_{t}\right|-k\right)}{(1+k)^{t}}\right\} Q(z) \\
& \quad-\bar{\lambda} M n(n-1) \cdots(n-t+1)\left(\frac{z}{k}\right)^{n} \\
& \quad \times\left(\alpha_{1} \cdot \alpha_{2} \cdots \alpha_{t}+\beta\left\{\frac{\left(\left|\alpha_{1}\right|-k\right)\left(\left|\alpha_{2}\right|-k\right) \cdots\left(\left|\alpha_{t}\right|-k\right)}{(1+k)^{t}}\right\}\right) \mid
\end{aligned}
$$

As $|P(z)|=|Q(z)|$ for $|z|=k$, that is, $M=\max _{|z|=k}|P(z)|=\max _{|z|=k}|Q(z)|$, by taking $\mu=1$ in Lemma 8 and using it to the polynomial $Q(z)$, we obtain the following:

$$
\begin{gathered}
\left|z^{t} D_{\alpha_{t}} \cdots D_{\alpha_{2}} D_{\alpha_{1}} Q(z)+\beta n(n-1) \cdots(n-t+1)\left\{\frac{\left(\left|\alpha_{1}\right|-k\right)\left(\left|\alpha_{2}\right|-k\right) \cdots\left(\left|\alpha_{t}\right|-k\right)}{(1+k)^{t}}\right\} Q(z)\right| \\
\quad<|\lambda| n(n-1) \cdots(n-t+1) \frac{M}{k^{n}}\left|\alpha_{1} \cdot \alpha_{2} \cdots \alpha_{t}+\beta\left\{\frac{\left(\left|\alpha_{1}\right|-k\right)\left(\left|\alpha_{2}\right|-k\right) \cdots\left(\left|\alpha_{t}\right|-k\right)}{(1+k)^{t}}\right\}\right| .
\end{gathered}
$$


Thus, taking the argument of $\lambda$ suitably, we obtain

$$
\begin{aligned}
\mid z^{t} D_{\alpha_{t}} \cdots D_{\alpha_{2}} D_{\alpha_{1}} Q(z)+\beta n(n-1) \cdots(n-t+1)\left\{\frac{\left(\left|\alpha_{1}\right|-k\right)\left(\left|\alpha_{2}\right|-k\right) \cdots\left(\left|\alpha_{t}\right|-k\right)}{(1+k)^{t}}\right\} Q(z) & \\
& -\bar{\lambda} M n(n-1) \cdots(n-t+1)\left(\frac{z}{k}\right)^{n} \\
& \times\left(\alpha_{1} \cdot \alpha_{2} \cdots \alpha_{t}+\beta\left\{\frac{\left(\left|\alpha_{1}\right|-k\right)\left(\left|\alpha_{2}\right|-k\right) \cdots\left(\left|\alpha_{t}\right|-k\right)}{(1+k)^{t}}\right\}\right) \mid \\
=\mid & \lambda \mid M n(n-1) \cdots(n-t+1) \\
& \times\left|\frac{z}{k}\right|^{n}\left|\alpha_{1} \cdot \alpha_{2} \cdots \alpha_{t}+\beta\left\{\frac{\left(\left|\alpha_{1}\right|-k\right)\left(\left|\alpha_{2}\right|-k\right) \cdots\left(\left|\alpha_{t}\right|-k\right)}{(1+k)^{t}}\right\}\right| \\
& -\mid z^{t} D_{\alpha_{t}} \cdots D_{\alpha_{2}} D_{\alpha_{1}} Q(z)+\beta n(n-1) \cdots(n-t+1) \\
& \times\left\{\frac{\left(\left|\alpha_{1}\right|-k\right)\left(\left|\alpha_{2}\right|-k\right) \cdots\left(\left|\alpha_{t}\right|-k\right)}{(1+k)^{t}}\right\} Q(z) \mid
\end{aligned}
$$

Using (28) in (27), we get for $|z|=1$ and $|\beta|<1$,

$$
\begin{aligned}
& \left|z^{t} D_{\alpha_{t}} \cdots D_{\alpha_{2}} D_{\alpha_{1}} P(z)+n(n-1) \cdots(n-t+1) \beta\left\{\frac{\left(\left|\alpha_{1}\right|-k\right)\left(\left|\alpha_{2}\right|-k\right) \cdots\left(\left|\alpha_{t}\right|-k\right)}{(1+k)^{t}}\right\} P(z)\right| \\
& \quad-|\lambda| n(n-1) \cdots(n-t+1) M\left|z^{t}+\beta\left\{\frac{\left(\left|\alpha_{1}\right|-k\right)\left(\left|\alpha_{2}\right|-k\right) \cdots\left(\left|\alpha_{t}\right|-k\right)}{(1+k)^{t}}\right\}\right| \\
& \leq|\lambda| n(n-1) \cdots(n-t+1) \frac{M}{k^{n}}\left|\alpha_{1} \cdot \alpha_{2} \cdots \alpha_{t}+\beta\left\{\frac{\left(\left|\alpha_{1}\right|-k\right)\left(\left|\alpha_{2}\right|-k\right) \cdots\left(\left|\alpha_{t}\right|-k\right)}{(1+k)^{t}}\right\}\right| \\
& \quad-\mid z^{t} D_{\alpha_{t}} \cdots D_{\alpha_{2}} D_{\alpha_{1}} Q(z)+n(n-1) \cdots(n-t+1) \\
& \quad \times \beta\left\{\frac{\left(\left|\alpha_{1}\right|-k\right)\left(\left|\alpha_{2}\right|-k\right) \cdots\left(\left|\alpha_{t}\right|-k\right)}{(1+k)^{t}}\right\} Q(z) \mid .
\end{aligned}
$$

That is,

$$
\begin{aligned}
\mid z^{t} D_{\alpha_{t}} \cdots D_{\alpha_{2}} D_{\alpha_{1}} P(z)+n(n-1) \cdots(n-t+1) \\
\quad \times \beta\left\{\frac{\left(\left|\alpha_{1}\right|-k\right)\left(\left|\alpha_{2}\right|-k\right) \cdots\left(\left|\alpha_{t}\right|-k\right)}{\left.(1+k)^{t}\right\} P(z) \mid}\right. \\
\quad+\mid z^{t} D_{\alpha_{t}} \cdots D_{\alpha_{2}} D_{\alpha_{1}} Q(z)+n(n-1) \cdots(n-t+1) \\
\quad \times \beta\left\{\frac{\left(\left|\alpha_{1}\right|-k\right)\left(\left|\alpha_{2}\right|-k\right) \cdots\left(\left|\alpha_{t}\right|-k\right)}{\left.(1+k)^{t}\right\} Q(z) \mid}\right. \\
\leq|\lambda|\left\{\left|\alpha_{1} \cdot \alpha_{2} \cdots \alpha_{t}+\beta\left\{\frac{\left(\left|\alpha_{1}\right|-k\right)\left(\left|\alpha_{2}\right|-k\right) \cdots\left(\left|\alpha_{t}\right|-k\right)}{(1+k)^{t}}\right\}\right| \cdot \frac{1}{k^{n}}\right. \\
\left.+\left|z^{t}+\beta\left\{\frac{\left(\left|\alpha_{1}\right|-k\right)\left(\left|\alpha_{2}\right|-k\right) \cdots\left(\left|\alpha_{t}\right|-k\right)}{(1+k)^{t}}\right\}\right|\right\} n(n-1) \cdots(n-t+1) M .
\end{aligned}
$$


Taking $|\lambda| \rightarrow 1$, we get

$$
\begin{aligned}
& \left|z^{t} D_{\alpha_{t}} \cdots D_{\alpha_{2}} D_{\alpha_{1}} P(z)+n(n-1) \cdots(n-t+1) \beta\left\{\frac{\left(\left|\alpha_{1}\right|-k\right)\left(\left|\alpha_{2}\right|-k\right) \cdots\left(\left|\alpha_{t}\right|-k\right)}{(1+k)^{t}}\right\} P(z)\right| \\
& \quad+\mid z^{t} D_{\alpha_{t}} \cdots D_{\alpha_{2}} D_{\alpha_{1}} Q(z)+n(n-1) \cdots(n-t+1) \\
& \quad \times \beta\left\{\frac{\left(\left|\alpha_{1}\right|-k\right)\left(\left|\alpha_{2}\right|-k\right) \cdots\left(\left|\alpha_{t}\right|-k\right)}{(1+k)^{t}}\right\} Q(z) \mid \\
& \leq\left\{\left|\alpha_{1} \cdot \alpha_{2} \cdots \alpha_{t}+\beta\left\{\frac{\left(\left|\alpha_{1}\right|-k\right)\left(\left|\alpha_{2}\right|-k\right) \cdots\left(\left|\alpha_{t}\right|-k\right)}{(1+k)^{t}}\right\}\right| \cdot \frac{1}{k^{n}}\right. \\
& \left.\quad+\left|z^{t}+\beta\left\{\frac{\left(\left|\alpha_{1}\right|-k\right)\left(\left|\alpha_{2}\right|-k\right) \cdots\left(\left|\alpha_{t}\right|-k\right)}{(1+k)^{t}}\right\}\right|\right\} n(n-1) \cdots(n-t+1) M .
\end{aligned}
$$

Then, by applying the maximum modulus principle for the polynomial $P(z)$ when $|k| \leq 1$,

$$
\max _{|z|=k}|P(z)| \leq \max _{|z|=1}|P(z)|
$$

This in conjunction with (29) and the argument of continuity gives the result.

Lemma 10 Let $H(z)$ be a polynomial of degree $n$ having all its zeros in $|z| \leq k, k \leq 1$, and let $G(z)$ be a polynomial of degree not exceeding that of $H(z)$. If $|G(z)| \leq|H(z)|$ for $|z|=k$, $k \leq 1$, then for all real or complex numbers $\alpha_{i}$ with $\left|\alpha_{i}\right| \geq k, i=1,2, \ldots, t$, and for any real or complex number $\beta$ with $|\beta| \leq 1$, and $|z|=1$, we have

$$
\begin{aligned}
& \mid z^{t} D_{\alpha_{t}} \cdots D_{\alpha_{2}} D_{\alpha_{1}} G(z)+n(n-1) \cdots(n-t+1) \\
& \quad \times \beta\left\{\frac{\left(\left|\alpha_{1}\right|-k\right)\left(\left|\alpha_{2}\right|-k\right) \cdots\left(\left|\alpha_{t}\right|-k\right)}{(1+k)^{t}}\right\} G(z) \mid \\
& \leq \mid z^{t} D_{\alpha_{t}} \cdots D_{\alpha_{2}} D_{\alpha_{1}} H(z)+n(n-1) \cdots(n-t+1) \\
& \quad \times \beta\left\{\frac{\left(\left|\alpha_{1}\right|-k\right)\left(\left|\alpha_{2}\right|-k\right) \cdots\left(\left|\alpha_{t}\right|-k\right)}{(1+k)^{t}}\right\} H(z) \mid .
\end{aligned}
$$

Proof of Lemma 10 For any real or complex number $\lambda$ with $|\lambda|<1$, we have $|\lambda G(z)|<$ $|G(z)| \leq|H(z)|$ for $|z|=k, k \leq 1$; therefore, by Rouche's theorem $H(z)-\lambda G(z)$ and $H(z)$ have the same number of zeros in $|z|<k$. Also, as $|G(z)| \leq|H(z)|$ for $|z|=k, k \leq 1$, any zero of $H(z)$ that lies on $|z|=k$ is also a zero of $G(z)$. Therefore, $H(z)-\lambda G(z)$ has all its zeros in the closed disk $|z| \leq k$. Therefore, using Lemma 6 with $\mu=1$, we have for every real or complex number $\alpha_{i}$ with $\left|\alpha_{i}\right| \geq k, i=1,2, \ldots, t, k \leq 1(1 \leq t<n)$ and $|z|=1$,

$$
\begin{aligned}
& \left|z^{t} D_{\alpha_{t}} \cdots D_{\alpha_{2}} D_{\alpha_{1}}(H(z)-\lambda G(z))\right| \\
& \quad \geq n(n-1) \cdots(n-t+1)\left\{\frac{\left(\left|\alpha_{1}\right|-k\right)\left(\left|\alpha_{2}\right|-k\right) \cdots\left(\left|\alpha_{t}\right|-k\right)}{(1+k)^{t}}\right\}|H(z)-\lambda G(z)| .
\end{aligned}
$$


Now, by a similar argument as that used in the proof of Lemma 7, for any real or complex number $\beta$ with $|\beta|<1$, we get

$$
\begin{aligned}
& \left|z^{t} D_{\alpha_{t}} \cdots D_{\alpha_{2}} D_{\alpha_{1}}(H(z)-\lambda G(z))\right| \\
& \quad \geq n(n-1) \cdots(n-t+1)\left\{\frac{\left(\left|\alpha_{1}\right|-k\right)\left(\left|\alpha_{2}\right|-k\right) \cdots\left(\left|\alpha_{t}\right|-k\right)}{(1+k)^{t}}\right\}|H(z)-\lambda G(z)| \\
& \quad>n(n-1) \cdots(n-t+1)|\beta|\left\{\frac{\left(\left|\alpha_{1}\right|-k\right)\left(\left|\alpha_{2}\right|-k\right) \cdots\left(\left|\alpha_{t}\right|-k\right)}{(1+k)^{t}}\right\}|H(z)-\lambda G(z)|
\end{aligned}
$$

for $|z|=1$, which implies

$$
\begin{aligned}
T(z)= & z^{t} D_{\alpha_{t}} \cdots D_{\alpha_{2}} D_{\alpha_{1}} H(z)-\lambda z^{t} D_{\alpha_{t}} \cdots D_{\alpha_{2}} D_{\alpha_{1}} G(z) \\
& +n(n-1) \cdots(n-t+1) \beta\left\{\frac{\left(\left|\alpha_{1}\right|-k\right)\left(\left|\alpha_{2}\right|-k\right) \cdots\left(\left|\alpha_{t}\right|-k\right)}{(1+k)^{t}}\right\} \\
& \times(H(z)-\lambda G(z)) \neq 0
\end{aligned}
$$

for $|z|=1$.

That is,

$$
\begin{aligned}
T(z)= & z^{t} D_{\alpha_{t}} \cdots D_{\alpha_{2}} D_{\alpha_{1}} H(z)+n(n-1) \cdots(n-t+1) \\
& \times \beta\left\{\frac{\left(\left|\alpha_{1}\right|-k\right)\left(\left|\alpha_{2}\right|-k\right) \cdots\left(\left|\alpha_{t}\right|-k\right)}{(1+k)^{t}}\right\} H(z) \\
& -\lambda\left[z^{t} D_{\alpha_{t}} \cdots D_{\alpha_{2}} D_{\alpha_{1}} G(z)+n(n-1) \cdots(n-t+1)\right. \\
& \left.\times \beta\left\{\frac{\left(\left|\alpha_{1}\right|-k\right)\left(\left|\alpha_{2}\right|-k\right) \cdots\left(\left|\alpha_{t}\right|-k\right)}{(1+k)^{t}}\right\} G(z)\right] \neq 0
\end{aligned}
$$

for $|z|=1$.

So, we conclude that

$$
\begin{aligned}
& \mid z^{t} D_{\alpha_{t}} \cdots D_{\alpha_{2}} D_{\alpha_{1}} H(z)+n(n-1) \cdots(n-t+1) \\
& \quad \times \beta\left\{\frac{\left(\left|\alpha_{1}\right|-k\right)\left(\left|\alpha_{2}\right|-k\right) \cdots\left(\left|\alpha_{t}\right|-k\right)}{(1+k)^{t}}\right\} H(z) \mid \\
& \geq \mid z^{t} D_{\alpha_{t}} \cdots D_{\alpha_{2}} D_{\alpha_{1}} G(z)+n(n-1) \cdots(n-t+1) \\
& \quad \times \beta\left\{\frac{\left(\left|\alpha_{1}\right|-k\right)\left(\left|\alpha_{2}\right|-k\right) \cdots\left(\left|\alpha_{t}\right|-k\right)}{(1+k)^{t}}\right\} G(z) \mid
\end{aligned}
$$

for $|z|=1$.

If (31) is not true, then there exists a point $z=\omega$ with $|\omega|=1$ such that

$$
\begin{aligned}
& \mid \omega^{t} D_{\alpha_{t}} \cdots D_{\alpha_{2}} D_{\alpha_{1}} H(\omega)+n(n-1) \cdots(n-t+1) \\
& \quad \times \beta\left\{\frac{\left(\left|\alpha_{1}\right|-k\right)\left(\left|\alpha_{2}\right|-k\right) \cdots\left(\left|\alpha_{t}\right|-k\right)}{(1+k)^{t}}\right\} H(\omega) \mid
\end{aligned}
$$




$$
\begin{array}{r}
<\mid \omega^{t} D_{\alpha_{t}} \cdots D_{\alpha_{2}} D_{\alpha_{1}} G(\omega)+n(n-1) \cdots(n-t+1) \\
\quad \times \beta\left\{\frac{\left(\left|\alpha_{1}\right|-k\right)\left(\left|\alpha_{2}\right|-k\right) \cdots\left(\left|\alpha_{t}\right|-k\right)}{(1+k)^{t}}\right\} G(\omega) \mid .
\end{array}
$$

Take

$$
\lambda=\frac{\omega^{t} D_{\alpha_{t}} \cdots D_{\alpha_{2}} D_{\alpha_{1}} H(\omega)+n(n-1) \cdots(n-t+1) \beta\left\{\frac{\left(\left|\alpha_{1}\right|-k\right)\left(\left|\alpha_{2}\right|-k\right) \cdots\left(\left|\alpha_{t}\right|-k\right)}{(1+k)^{t}}\right\} H(\omega)}{\omega^{t} D_{\alpha_{t}} \cdots D_{\alpha_{2}} D_{\alpha_{1}} G(\omega)+n(n-1) \cdots(n-t+1) \beta\left\{\frac{\left(\left|\alpha_{1}\right|-k\right)\left(\left|\alpha_{2}\right|-k\right) \cdots\left(\left|\alpha_{t}\right|-k\right)}{(1+k)^{t}}\right\} G(\omega)},
$$

then $|\lambda|<1$, and with this choice of $\lambda$, we have from (30) $T(\omega)=0$ for $|\omega|=1$. But this contradicts the fact that $T(z) \neq 0$ for $|z|=1$. For $\beta$ with $|\beta|=1$, (31) follows by continuity. This completes the proof of Lemma 10.

\section{Proof of the theorem}

Since the polynomial $P(z)$ has no zeros in the disk $|z|<k$, and therefore, if $m=$ $\min _{|z|=k}|P(z)|$, then $m \leq|P(z)|$ for $|z| \leq k$. If $\lambda$ is any real or complex number with $|\lambda|<1$, we have

$$
|\lambda m|<m \leq|P(z)| \text { for }|z|=k
$$

Thus, by Rouche's theorem, the polynomial $G(z)=P(z)-\lambda m$ does not vanish in $|z|<k$. Consequently, the polynomial

$$
H(z):=\left(\frac{z}{k}\right)^{n} \overline{G\left(\frac{k^{2}}{\bar{z}}\right)}=Q(z)-\bar{\lambda} m\left(\frac{z}{k}\right)^{n}
$$

has all its zeros in $|z| \leq k$, where $Q(z)=\left(\frac{z}{k}\right)^{n} \overline{P\left(\frac{k^{2}}{\bar{z}}\right)}$, and also we have $|G(z)|=|H(z)|$ for $|z|=k$.

Applying Lemma 10 to the polynomials $H(z)$ and $G(z)$, we have

$$
\begin{aligned}
& \left|z^{t} D_{\alpha_{t}} \cdots D_{\alpha_{2}} D_{\alpha_{1}} G(z)+n(n-1) \cdots(n-t+1) \beta\left\{\frac{\left(\left|\alpha_{1}\right|-k\right)\left(\left|\alpha_{2}\right|-k\right) \cdots\left(\left|\alpha_{t}\right|-k\right)}{(1+k)^{t}}\right\} G(z)\right| \\
& \leq \mid z^{t} D_{\alpha_{t}} \cdots D_{\alpha_{2}} D_{\alpha_{1}} H(z)+n(n-1) \cdots(n-t+1) \\
& \quad \times \beta\left\{\frac{\left(\left|\alpha_{1}\right|-k\right)\left(\left|\alpha_{2}\right|-k\right) \cdots\left(\left|\alpha_{t}\right|-k\right)}{(1+k)^{t}}\right\} H(z) \mid
\end{aligned}
$$

for every real or complex number $\alpha_{i}$ with $\left|\alpha_{i}\right| \geq k, i=1,2, \ldots, t$, and for any real or complex number $\beta$ with $|\beta| \leq 1$ and $|z|=1$.

On substituting $G(z)$ and $H(z)$ in the above inequality, we obtain the following for every real or complex number $\alpha_{i}$ with $\left|\alpha_{i}\right| \geq k, i=1,2, \ldots, t$, and for any real or complex number $\beta$ with $|\beta| \leq 1$ and $|z|=1$,

$$
\begin{aligned}
& \mid z^{t} D_{\alpha_{t}} \cdots D_{\alpha_{2}} D_{\alpha_{1}} P(z)+\beta n(n-1) \cdots(n-t+1)\left\{\frac{\left(\left|\alpha_{1}\right|-k\right)\left(\left|\alpha_{2}\right|-k\right) \cdots\left(\left|\alpha_{t}\right|-k\right)}{(1+k)^{t}}\right\} P(z) \\
& -\lambda m n(n-1) \cdots(n-t+1)\left(z^{t}+\beta\left\{\frac{\left(\left|\alpha_{1}\right|-k\right)\left(\left|\alpha_{2}\right|-k\right) \cdots\left(\left|\alpha_{t}\right|-k\right)}{(1+k)^{t}}\right\}\right) \mid
\end{aligned}
$$




$$
\begin{aligned}
\leq & \mid z^{t} D_{\alpha_{t}} \cdots D_{\alpha_{2}} D_{\alpha_{1}} Q(z)+\beta n(n-1) \cdots(n-t+1) \\
& \times\left\{\frac{\left(\left|\alpha_{1}\right|-k\right)\left(\left|\alpha_{2}\right|-k\right) \cdots\left(\left|\alpha_{t}\right|-k\right)}{(1+k)^{t}}\right\} Q(z) \\
& -\bar{\lambda} m n(n-1) \cdots(n-t+1)\left(\frac{z}{k}\right)^{n} \\
& \times\left(\alpha_{1} \cdot \alpha_{2} \cdots \alpha_{t}+\beta\left\{\frac{\left(\left|\alpha_{1}\right|-k\right)\left(\left|\alpha_{2}\right|-k\right) \cdots\left(\left|\alpha_{t}\right|-k\right)}{(1+k)^{t}}\right\}\right) .
\end{aligned}
$$

Since all the zeros of $Q(z)$ lie in $|z| \leq k$ and $|P(z)|=|Q(z)|$ for $|z|=k$, therefore, by applying Lemma 7 to $Q(z)$, we have

$$
\begin{aligned}
& \mid z^{t} D_{\alpha_{t}} \cdots D_{\alpha_{2}} D_{\alpha_{1}} Q(z)+\beta n(n-1) \cdots(n-t+1) \\
& \times\left\{\frac{\left(\left|\alpha_{1}\right|-k\right)\left(\left|\alpha_{2}\right|-k\right) \cdots\left(\left|\alpha_{t}\right|-k\right)}{(1+k)^{t}}\right\} Q(z) \mid \\
& \geq n(n-1) \cdots(n-t+1) \\
& \times \frac{1}{k^{n}}\left|\alpha_{1} \cdot \alpha_{2} \cdots \alpha_{t}+\beta\left\{\frac{\left(\left|\alpha_{1}\right|-k\right)\left(\left|\alpha_{2}\right|-k\right) \cdots\left(\left|\alpha_{t}\right|-k\right)}{(1+k)^{t}}\right\}\right| \min _{|z|=k}|Q(z)| \\
& =n(n-1) \cdots(n-t+1) \frac{1}{k^{n}}\left|\alpha_{1} \cdot \alpha_{2} \cdots \alpha_{t}+\beta\left\{\frac{\left(\left|\alpha_{1}\right|-k\right)\left(\left|\alpha_{2}\right|-k\right) \cdots\left(\left|\alpha_{t}\right|-k\right)}{(1+k)^{t}}\right\}\right| m \text {. }
\end{aligned}
$$

Then, for an appropriate choice of the argument of $\lambda$, we get

$$
\begin{aligned}
\mid z^{t} D_{\alpha_{t}} \cdots D_{\alpha_{2}} D_{\alpha_{1}} Q(z)+\beta n(n-1) \cdots(n-t+1) & \\
\quad & \times\left\{\frac{\left(\left|\alpha_{1}\right|-k\right)\left(\left|\alpha_{2}\right|-k\right) \cdots\left(\left|\alpha_{t}\right|-k\right)}{(1+k)^{t}}\right\} Q(z) \\
& -\bar{\lambda} m n(n-1) \cdots(n-t+1)\left(\frac{z}{k}\right)^{n} \\
& \times\left(\alpha_{1} \cdot \alpha_{2} \cdots \alpha_{t}+\beta\left\{\frac{\left(\left|\alpha_{1}\right|-k\right)\left(\left|\alpha_{2}\right|-k\right) \cdots\left(\left|\alpha_{t}\right|-k\right)}{(1+k)^{t}}\right\}\right) \mid \\
= & \mid z^{t} D_{\alpha_{t}} \cdots D_{\alpha_{2}} D_{\alpha_{1}} Q(z)+\beta n(n-1) \cdots(n-t+1) \\
\quad & \times\left\{\frac{\left(\left|\alpha_{1}\right|-k\right)\left(\left|\alpha_{2}\right|-k\right) \cdots\left(\left|\alpha_{t}\right|-k\right)}{(1+k)^{t}}\right\} Q(z) \mid \\
& -|\lambda| m n(n-1) \cdots(n-t+1) \\
\quad & \times \frac{1}{k^{n}}\left|\alpha_{1} \cdot \alpha_{2} \cdots \alpha_{t}+\beta\left\{\frac{\left(\left|\alpha_{1}\right|-k\right)\left(\left|\alpha_{2}\right|-k\right) \cdots\left(\left|\alpha_{t}\right|-k\right)}{(1+k)^{t}}\right\}\right|
\end{aligned}
$$

for $|z|=1$.

Then combining the right-hand side of (32) and (33), we rewrite (32) as

$$
\begin{aligned}
& \left|z^{t} D_{\alpha_{t}} \cdots D_{\alpha_{2}} D_{\alpha_{1}} P(z)+\beta n(n-1) \cdots(n-t+1)\left\{\frac{\left(\left|\alpha_{1}\right|-k\right)\left(\left|\alpha_{2}\right|-k\right) \cdots\left(\left|\alpha_{t}\right|-k\right)}{(1+k)^{t}}\right\} P(z)\right| \\
& -|\lambda| m n(n-1) \cdots(n-t+1)\left|z^{t}+\beta\left\{\frac{\left(\left|\alpha_{1}\right|-k\right)\left(\left|\alpha_{2}\right|-k\right) \cdots\left(\left|\alpha_{t}\right|-k\right)}{(1+k)^{t}}\right\}\right|
\end{aligned}
$$


Singh et al. Journal of Inequalities and Applications 2013, 2013:183

Page 17 of 19

http://www.journalofinequalitiesandapplications.com/content/2013/1/183

$$
\begin{aligned}
\leq & \mid z^{t} D_{\alpha_{t}} \cdots D_{\alpha_{2}} D_{\alpha_{1}} Q(z)+\beta n(n-1) \cdots(n-t+1) \\
& \times\left\{\frac{\left(\left|\alpha_{1}\right|-k\right)\left(\left|\alpha_{2}\right|-k\right) \cdots\left(\left|\alpha_{t}\right|-k\right)}{(1+k)^{t}}\right\} Q(z) \mid \\
& -|\lambda| m n(n-1) \cdots(n-t+1) \\
& \times \frac{1}{k^{n}}\left|\alpha_{1} \cdot \alpha_{2} \cdots \alpha_{t}+\beta\left\{\frac{\left(\left|\alpha_{1}\right|-k\right)\left(\left|\alpha_{2}\right|-k\right) \cdots\left(\left|\alpha_{t}\right|-k\right)}{(1+k)^{t}}\right\}\right| \quad \text { for }|z|=1 .
\end{aligned}
$$

Equivalently,

$$
\begin{aligned}
& \left|z^{t} D_{\alpha_{t}} \cdots D_{\alpha_{2}} D_{\alpha_{1}} P(z)+\beta n(n-1) \cdots(n-t+1)\left\{\frac{\left(\left|\alpha_{1}\right|-k\right)\left(\left|\alpha_{2}\right|-k\right) \cdots\left(\left|\alpha_{t}\right|-k\right)}{(1+k)^{t}}\right\} P(z)\right| \\
& \leq \mid z^{t} D_{\alpha_{t}} \cdots D_{\alpha_{2}} D_{\alpha_{1}} Q(z)+\beta n(n-1) \cdots(n-t+1) \\
& \quad \times\left\{\frac{\left(\left|\alpha_{1}\right|-k\right)\left(\left|\alpha_{2}\right|-k\right) \cdots\left(\left|\alpha_{t}\right|-k\right)}{(1+k)^{t}}\right\} Q(z) \mid \\
& \quad-|\lambda| m n(n-1) \cdots(n-t+1) \\
& \quad \times\left\{\frac{1}{k^{n}}\left|\alpha_{1} \cdot \alpha_{2} \cdots \alpha_{t}+\beta\left\{\frac{\left(\left|\alpha_{1}\right|-k\right)\left(\left|\alpha_{2}\right|-k\right) \cdots\left(\left|\alpha_{t}\right|-k\right)}{(1+k)^{t}}\right\}\right|\right. \\
& \left.\quad-\left|z^{t}+\beta\left\{\frac{\left(\left|\alpha_{1}\right|-k\right)\left(\left|\alpha_{2}\right|-k\right) \cdots\left(\left|\alpha_{t}\right|-k\right)}{(1+k)^{t}}\right\}\right|\right\} .
\end{aligned}
$$

As $|\lambda| \rightarrow 1$, we have

$$
\begin{aligned}
& \left|z^{t} D_{\alpha_{t}} \cdots D_{\alpha_{2}} D_{\alpha_{1}} P(z)+\beta n(n-1) \cdots(n-t+1)\left\{\frac{\left(\left|\alpha_{1}\right|-k\right)\left(\left|\alpha_{2}\right|-k\right) \cdots\left(\left|\alpha_{t}\right|-k\right)}{(1+k)^{t}}\right\} P(z)\right| \\
& \leq \mid z^{t} D_{\alpha_{t}} \cdots D_{\alpha_{2}} D_{\alpha_{1}} Q(z)+\beta n(n-1) \cdots(n-t+1) \\
& \quad \times\left\{\frac{\left(\left|\alpha_{1}\right|-k\right)\left(\left|\alpha_{2}\right|-k\right) \cdots\left(\left|\alpha_{t}\right|-k\right)}{(1+k)^{t}}\right\} Q(z) \mid \\
& \quad-m n(n-1) \cdots(n-t+1) \\
& \quad \times\left\{\frac{1}{k^{n}}\left|\alpha_{1} \cdot \alpha_{2} \cdots \alpha_{t}+\beta\left\{\frac{\left(\left|\alpha_{1}\right|-k\right)\left(\left|\alpha_{2}\right|-k\right) \cdots\left(\left|\alpha_{t}\right|-k\right)}{(1+k)^{t}}\right\}\right|\right. \\
& \left.\quad-\left|z^{t}+\beta\left\{\frac{\left(\left|\alpha_{1}\right|-k\right)\left(\left|\alpha_{2}\right|-k\right) \cdots\left(\left|\alpha_{t}\right|-k\right)}{(1+k)^{t}}\right\}\right|\right\} .
\end{aligned}
$$

It implies that for every real or complex number $\beta$ with $|\beta| \leq 1$ and $|z|=1$,

$$
\begin{aligned}
& 2 \mid z^{t} D_{\alpha_{t}} \cdots D_{\alpha_{2}} D_{\alpha_{1}} P(z)+\beta n(n-1) \cdots(n-t+1) \\
& \quad \times\left\{\frac{\left(\left|\alpha_{1}\right|-k\right)\left(\left|\alpha_{2}\right|-k\right) \cdots\left(\left|\alpha_{t}\right|-k\right)}{(1+k)^{t}}\right\} P(z) \mid \\
& \leq \mid z^{t} D_{\alpha_{t}} \cdots D_{\alpha_{2}} D_{\alpha_{1}} P(z)+\beta n(n-1) \cdots(n-t+1) \\
& \quad \times\left\{\frac{\left(\left|\alpha_{1}\right|-k\right)\left(\left|\alpha_{2}\right|-k\right) \cdots\left(\left|\alpha_{t}\right|-k\right)}{(1+k)^{t}}\right\} P(z) \mid
\end{aligned}
$$




$$
\begin{aligned}
& +\mid z^{t} D_{\alpha_{t}} \cdots D_{\alpha_{2}} D_{\alpha_{1}} Q(z)+\beta n(n-1) \cdots(n-t+1) \\
& \times\left\{\frac{\left(\left|\alpha_{1}\right|-k\right)\left(\left|\alpha_{2}\right|-k\right) \cdots\left(\left|\alpha_{t}\right|-k\right)}{(1+k)^{t}}\right\} Q(z) \mid \\
& -m n(n-1) \cdots(n-t+1) \\
& \times\left\{\frac{1}{k^{n}}\left|\alpha_{1} \cdot \alpha_{2} \cdots \alpha_{t}+\beta\left\{\frac{\left(\left|\alpha_{1}\right|-k\right)\left(\left|\alpha_{2}\right|-k\right) \cdots\left(\left|\alpha_{t}\right|-k\right)}{(1+k)^{t}}\right\}\right|\right. \\
& \left.-\left|z^{t}+\beta\left\{\frac{\left(\left|\alpha_{1}\right|-k\right)\left(\left|\alpha_{2}\right|-k\right) \cdots\left(\left|\alpha_{t}\right|-k\right)}{(1+k)^{t}}\right\}\right|\right\} .
\end{aligned}
$$

This in conjunction with Lemma 9 gives, for $|\beta| \leq 1$ and $|z|=1$,

$$
\begin{aligned}
& 2 \mid z^{t} D_{\alpha_{t}} \cdots D_{\alpha_{2}} D_{\alpha_{1}} P(z)+\beta n(n-1) \cdots(n-t+1) \\
& \quad \times\left\{\frac{\left(\left|\alpha_{1}\right|-k\right)\left(\left|\alpha_{2}\right|-k\right) \cdots\left(\left|\alpha_{t}\right|-k\right)}{(1+k)^{t}}\right\} P(z) \mid \\
& \leq n(n-1) \cdots(n-t+1)\left\{\frac{1}{k^{n}}\left|\alpha_{1} \cdot \alpha_{2} \cdots \alpha_{t}+\beta\left\{\frac{\left(\left|\alpha_{1}\right|-k\right)\left(\left|\alpha_{2}\right|-k\right) \cdots\left(\left|\alpha_{t}\right|-k\right)}{(1+k)^{t}}\right\}\right|\right. \\
&\left.+\left|z^{t}+\beta\left\{\frac{\left(\left|\alpha_{1}\right|-k\right)\left(\left|\alpha_{2}\right|-k\right) \cdots\left(\left|\alpha_{t}\right|-k\right)}{(1+k)^{t}}\right\}\right|\right\} \max _{|z|=1}|P(z)| \\
&-n(n-1) \cdots(n-t+1)\left\{\frac{1}{k^{n}}\left|\alpha_{1} \cdot \alpha_{2} \cdots \alpha_{t}+\beta\left\{\frac{\left(\left|\alpha_{1}\right|-k\right)\left(\left|\alpha_{2}\right|-k\right) \cdots\left(\left|\alpha_{t}\right|-k\right)}{(1+k)^{t}}\right\}\right|\right. \\
&\left.-\left|z^{t}+\beta\left\{\frac{\left(\left|\alpha_{1}\right|-k\right)\left(\left|\alpha_{2}\right|-k\right) \cdots\left(\left|\alpha_{t}\right|-k\right)}{(1+k)^{t}}\right\}\right|\right\} \min _{|z|=k}|P(z)| .
\end{aligned}
$$

This completes the proof of the theorem.

\section{Competing interests}

The authors declare that they have no competing interests.

\section{Authors' contributions}

GS and AL studied the related literature under the supervision of WMS and jointly developed the idea and drafted the manuscript. GS made the text file in Latex and communicated the manuscript. GS also revised it as per the directions of the referee under the guidance of WMS. All three authors read and approved the final manuscript.

\section{Author details}

${ }^{1}$ Bharathiar University, Coimbatore, Tamil Nadu 641046, India. ²Department of Mathematics, Kashmir University, Srinagar, 190006, India. ${ }^{3}$ Department of Mathematics, National Institute of Technology, Kashmir, 190006, India.

\section{Acknowledgements}

The authors are grateful to the referee for useful comments.

\section{Received: 24 August 2012 Accepted: 12 March 2013 Published: 17 April 2013}

\section{References}

1. Lax, PD: Proof of a conjecture of P. Erdös on the derivative of a polynomial. Bull. Am. Math. Soc. 50, 509-513 (1944)

2. Aziz, A, Dawood, QM: Inequalities for a polynomial and its derivative. J. Approx. Theory 54(3), 306-313 (1988)

3. Dewan, KK, Hans, S: Generalization of certain well-known polynomial inequalities. J. Math. Anal. Appl. 363(1), 38-41 (2010)

4. Aziz, A, Shah, WM: Inequalities for the polar derivative of a polynomial. Indian J. Pure Appl. Math. 29(2), 163-173 (1998)

5. Aziz, A, Mohammad Shah, W: Some inequalities for the polar derivative of a polynomial. Proc. Indian Acad. Sci. Math. Sci. 107(3), 263-270 (1997)

6. Liman, A, Mohapatra, RN, Shah, WM: Inequalities for the polar derivative of a polynomial. Complex Anal. Oper. Theory 6. 1199-1209 (2012). doi:10.1007/s11785-010-0120-3

7. Marden, M: Geometry of Polynomials, 2nd edn. Math. Surveys, vol. 3. Am. Math. Soc., Providence (1966) 
8. Aziz, A: A new proof of Laguerre's theorem about the zeros of polynomials. Bull. Aust. Math. Soc. 33, 131-138 (1986)

9. Chan, TN, Malik, MA: On Erdös-Lax theorem. Proc. Indian Acad. Sci. Math. Sci. 92(3), 191-193 (1983)

doi:10.1186/1029-242X-2013-183

Cite this article as: Singh et al.: A generalized inequality for the polar derivative of a polynomial. Journal of Inequalities and Applications 2013 2013:183.

Submit your manuscript to a SpringerOpen ${ }^{\odot}$ journal and benefit from:

- Convenient online submission

Rigorous peer review

- Immediate publication on acceptance

- Open access: articles freely available online

- High visibility within the field

- Retaining the copyright to your article

Submit your next manuscript at springeropen.com 